The results of our study could afford the basis of research regarding the use of natural products and their inclusion complexes as anticancer agents and the shift to targeted therapy with higher efficacy and limited toxicity.

Acknowledgements This Research Project is co-financed by Greece and EU (European Social Fund) through the Operational Program «Human Resources Development, Education and Lifelong Learning 2014-2020» and the Program titled 'Support for Post-doctoral Researchers- cycle B' (MIS 5033021), implemented by IKY (State Scholarships Foundation).

\section{L-ASPARAGINASE ACTIVITY MONITORING IN PAEDIATRIC HEMATOLOGICAL CANCER PATIENTS - CROATIAN EXPERIENCES}

Jasna Lenicek Krleza, Ana Katusic Bojanac*, D Buljan, Izabela Kranjcec, Višnja Armanda, Gordana Jakovljevic. Department of Medical Biology, University of Zagreb, School of Medicine, Zagreb, Croatia; Centre of Excellence for Reproductive and Regenerative Medicine, University of Zagreb School of Medicine, Zagreb, Croatia

\subsection{6/archdischild-2021-europaediatrics.317}

L-asparaginase has been an essential component of paediatricbased multiagent therapy for children diagnosed with haematological cancer.

Drug's effect is based on depletion of asparagine in the circulatory system thus depriving unmatured malignant cells of amino acid. However, some children are faced with silent inactivation or, in worst cases, an allergic reaction to the drug and is recommended to monitor drug activity levels in order to detect these patients and modify the therapy. We had set up the first laboratory for asparaginase-activity testing in Croatia at the Department of Laboratory diagnostics, Children's Hospital Zagreb. Our aim is to present the first experiences in asparaginase monitoring from March 2018 till October 2020 from the 2 pediatric centers (Zagreb and Split).

Children between 1 and 18 years old with diagnosed pediatric acute lymphoblastic leukemia (ALL) or lymphoma (NHL) were included in the prospective L-asparaginase study. Blood samples were collected prior to and during treatment. Serum was separated and frozen prior to shipping. Over 100 samples were processed, among which some were repeatedly measured in order to determine the inter-day precision due to sample thawing. Activities of three commercially available drugs, E. coli-derived asparaginase or Erwinia chrysanthemi asparaginase or E.coli pegylated, PEG-asparaginase were measured in intervals as recommended. Laboratory measurements used plate reader-based indooxine method where L-aspartic beta-hydroxamate (AHA) was a substrate for the enzyme. The reading time was optimized to ensure the best results.

41 patients (38 ALL and 3 lymphoma cases) were included in the study. Among thirty native E. coli asparaginase-treated patients, three (10\%) of them developed an allergy and four (13\%) of them showed silent inactivation.

Patients without hypersensitivity to native drug had serum median trough levels of $281,3 \mathrm{U} / \mathrm{L}$ after $48 \mathrm{~h}$. Six patients were treated with Erwinia asparaginase; one developed an allergy and none silent inactivation while others had median through levels 90,3 U/L after $48 \mathrm{~h}$. The PEG asparaginase therapy was given to 4 patients where median trough decline was observed $(938,4 \mathrm{U} / \mathrm{L}, 479,5 \mathrm{U} / \mathrm{L}, 170,3 \mathrm{U} / \mathrm{L}$ for 7,14 or 21 days, respectively).

Serum samples were stabile after second thawing as were no significant difference in asparaginase level after re-testing. Additionally, the absorbance results showed no significant difference between the 10 minutes reading intervals.

Here we conclude that therapeutic drug monitoring is important for pediatric patients with hematological cancer. The spectrophotometric-based method showed precise performance and has been added to our routine clinical protocols.

\section{HAIRY POLYP OF THE NASOPHARYNX - THE IMPORTANCE OF TEAM WORK}

Jelena Roganovic*, Valkovic Zuic Petra, Velepic Marko, Roganovic Jelena. Clinical Hospital Centre Rijeka

10.1136/archdischild-2021-europaediatrics.318

Hairy polyps are rare benign congenital dermoid tumors consisting of both mesodermal and ectodermal elements. They appear as soft, fleshy, pedunculated masses typically located in nasopharynx or oropharynx, usually at birth or soon after birth. Clinical manifestations include respiratory distress, shortness of breath, cyanosis, and difficulties with oral intake. On histology, hairy polyps comprise an outer keratinizing squamous epithelium with adnexal tissue, including hair follicles, and central fibroadipose and cartilaginous tissue. The imaging of choice is magnetic resonance imaging (MRI). MRI is helpful in characterizing the lesions and providing preoperative diagnosis. Further, it delineates the site of origin of these lesions, which can facilitate surgical planning and exclude an intracranial component. This is of the major importance if trans-nasal approach with endoscopy is planned in order to avoid injury to critical structures, such as the internal carotid artery.

We present a case of a 6-week-old full-term female baby presented with intermittent upper airway obstruction. On otorhinolaryngologic examination, an oval, smooth mass was visible in the left side of oropharynx, appeared to descend from the lateral wall of nasopharynx. MRI revealed a lobular mass $10 \times 4.8 \mathrm{~mm}$ on the left nasopharynx, spanning from torus tubarius to pharyngeal recess. Four days later a complete surgical excision was performed. On histology the lesion demonstrated adnexal skin structures overlying mesenchymal tissue, comprised mostly of fat and cartilaginous nodules. Two smaller parts of $0.4 \mathrm{~cm}$ in diameter were covered with stratified squamous epithelium and consisted of muscle cells and seromucous glands. The findings were consistent with the hairy polyp. MRI repeated after 2 months was normal. At 1 year follow-up, the child is in a good condition and without breathing or oral intake difficulties.

This case emphasizes the importance of multidisciplinary approach in neonates with nasopharyngeal masses, in order to provide timely and accurate diagnosis as well as the optimal treatment. 